\title{
AUGMENTING A RUGGED STANDARD DJ TURNTABLE WITH A TANGIBLE INTERFACE FOR MUSIC BROWSING AND PLAYBACK MANIPULATION
}

\author{
Andreas Pabst, Roger Walk \\ University of Ulm \\ \{andreas.pabst, roger.walk\}@uni-ulm.de
}

Keywords: Tangible Interface, Human-Machine Interaction, Physical Computing, Augmented Reality

\begin{abstract}
TIMBAP is a new solution for the digital disc jockey (DJ) that provides a rugged tangible interface for music browsing and playback manipulation. Like many others it is based on an acoustic timecode signal recorded to vinyl records. In contrast to existing digital solutions however, it completely releases the DJ from mouse, keyboard and monitor. Instead it relies on physical interaction with the standard club turntable only. The rotation of the turntable serves as a means of scrolling through the music collection automatically. The user stays in control though as this can always be overridden by a manual intervention like holding the record or winding it back. In order to provide goaloriented search, we also support a direct absolute positioning using the tone arm.
\end{abstract}

\section{Motivation}

A disc jockey (DJ) plays music to an audience in different environments which can be a dancefloor, a bar, a fashion show or any other public or private setting. Until today many DJs refrain from all digital media (especially CDs) for their practice and stick with vinyl records and record players instead. There are many reasons for this behaviour including the need to stand out from the crowd. The richly nuanced natural interaction possibilities form the most important argument in favour of turntables though. Also the preference is very much connected to the success story of the Technics SL-1200 model. Despite its historic age of 35 years this model is still installed in nearly all dance clubs worldwide. Many of the so-called turntablists who use the turntable as a musical instrument will insist on using only the Technics SL-1200 due to the great tactile feel [6]. Furthermore it has proven robustness to an extraordinary degree, as some of the first models are said to be doing their duty until today.

This is why in 2002 Stanton Magnetics decided to build a new digital DJing system called Final Scratch that can be controlled using a regular turntable. They equipped the customer with special vinyl records containing an acoustic timecode signal. The timecode is constantly read from the incoming audio signal and mapped to playback velocity and absolute position of a digital audio file. This way DJs are enabled to manipulate the playback of audio files in exactly the same manner as they would handle a regular record. Hard-won technical skills like scratching or beatmatching can thus be applied to the digital domain without any loss. However, none of the existing systems goes beyond the plain standard WIMP interface for the DJs' other duty, which many consider to be even more important: music selection.

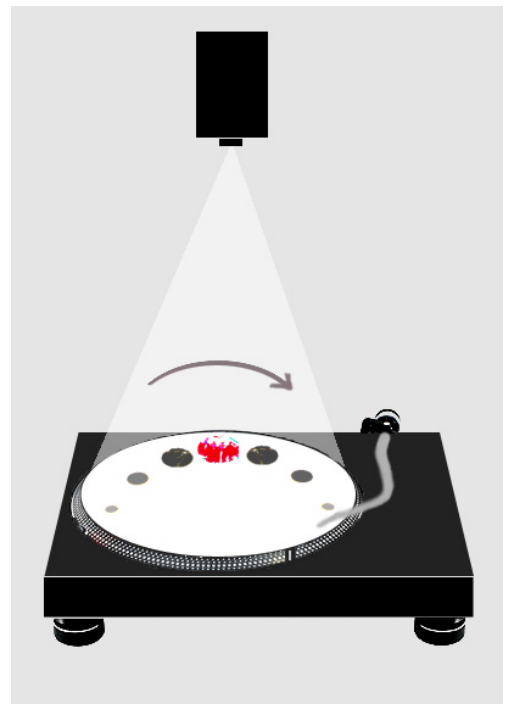

Figure 1: The graphical representation of the music browsing task is projected onto the turntable platter

We claim to have enhanced the user interface of digital DJing systems by using the common physical interaction patterns a DJ is used to [5] not only for playback manipulation but for music browsing and selection as well. The standard turntable becomes a complete tangible interface for digital DJs. It already provides rich and fine-grained input possibilities the turntablists have taken advantage of ever since. On the output side however, only auditory feedback can be presented to the user. This might be sufficient for musical expression tasks like scratching, for the stateful music browsing and selection task though, a graphical representation can effectively not be avoided. Hence we decided to augment the turntable with a fully operational tangible interface, so it can serve as a controlling device 
and as a graphical display at the same time. As depicted in figure 1 this was achieved by projecting a graphical representation of the current state onto the timecode record spinning on the record platter. For a luminous and sharp display with high colour fidelity the record should be made of white vinyl preferably. Concerning the musical expression task of DJing, projection onto a standard turntable within a digital DJing system has already been proposed by Andersen. He planned to project a circular waveform. [1]

In section 2 we will analyze the traditional way of music selection by examining interviews of active DJs in order to extract requirements for TIMBAP. We will then describe our idea of an aimless automatic browsing mechanism in section 3. As a goal-oriented search mechanism is needed as well we propose to enrich the interface with switchable tag cloud positioning in section 4 and 5 . In the final section 6 we will conclude our achievements and goals for TIMBAP.

\section{Analysis of the music selection task}

In order to determine the users' requirements for the use case music selection we interviewed more than 160 active DJs for electronic and/or dance music. Most of them are semi-professionals or hobby DJs. In general, a DJ will begin searching his record bag for the next song after he has started a record and put back the one he played before. Depending on the length of the songs the timeframe for browsing the collection and selecting a record can be harshly limited. According to 48 percent of the participants between 30 and 60 seconds are usually spent searching for the next record. We also found out that DJs will look for a specific record in most cases, but only 12 percent disclaimed that they ever browse their collection aimlessly. This convinced us to include both an automatic browsing and a search mechanism. Concerning goal-oriented search the key question is what information the user is able to provide about the record that he has in mind. The results in table 1 show the reliability of visual anchors provided by cover art.

\begin{tabular}{|l|l|l|l|l}
\hline artist & title & label & cover art & place in bag \\
\hline $61,2 \%$ & $46,1 \%$ & $59,2 \%$ & $\mathbf{8 6 , 2} \%$ & $66,45 \%$ \\
\hline
\end{tabular}

Table 1: What DJs have an idea of in a goal-oriented search.

A similar finding supports this: 92 percent of our interviewees approve that they recognize a record by its cover art when browsing for a specific one. This led us to the rather obvious idea of representing the digital audio files not purely textual, but as record items in the graphical representation - moving geometrical objects rendered with the corresponding cover art as a texture.

\section{Aimless browsing and music selection}

For TIMBAP we wanted the automatic turntable rotation to be translated to an effortless browsing of the music collection. This can be useful when the DJ has not yet a record in mind. To do so, the DJ would simply start the turntable and watch the record items pass by. Halting the turntable would pause the audio signal and thus pause the browsing. As a mapping between the circular rotation of the turntable and the horizontal scrolling of the record items we have adopted the conveyor belt metaphor [7]. This visualization metaphor presents items as a list on a conveyor belt. In our case the turntable acts as a gear wheel to manually or automatically drive, stop and rewind the belt and thus the items. This affordance is supposed to be rather obvious for most users.

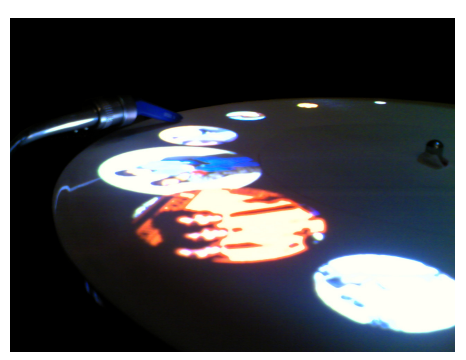

Figure 2: conveyor belt of music symbols

The browsing ends when a record item is to be selected. Unfortunately, the turntable interface does not provide any means to issue a direct command. To overcome this shortage, we decided to scale record items the bigger, the closer they are to the center of the platter. You can see this in figure 1. It is always the largest item that is assumed to be in focus. To actually select it for playback the record item has to be kept in focus for a certain time period. It will then grow to the full size of the turntable to visually indicate that it is being activated. When the full size is reached it is active and the DJ can start the playback by simply releasing the record. In an analogous manner the full-sized record item starts to shrink again towards its regular size when the turntable is halted for some time. The DJ can then activate the automatic browsing again by releasing the record.

\section{Tag cloud positioning for navigation}

In most cases however the DJ searches for a specific record. At this point you might think we would regret having eliminated keyboard and mouse. On closer inspection however, the turntable is equipped with some sort of pointing device: the tone arm. DJs are used to handle it precisely and rapidly from their skipping through heaps of mediocre records in the record shops. Unfortunately with an unsorted music collection the location of a certain audio file in the groove can only be guessed. This is why with TIMBAP the music collection is always sorted by a criterion. This might be artist name, label name, style, predominant colour in the art work etc. If the record items are distributed linearly over the full length of the timecode, a music piece for example by Mozart will hence appear when the timecode record is around half played. The DJ can set the tone arm to the middle and then subsequently adjust it by resetting or spinning forward or backward. He will soon come across his favourite Mozart piece. 


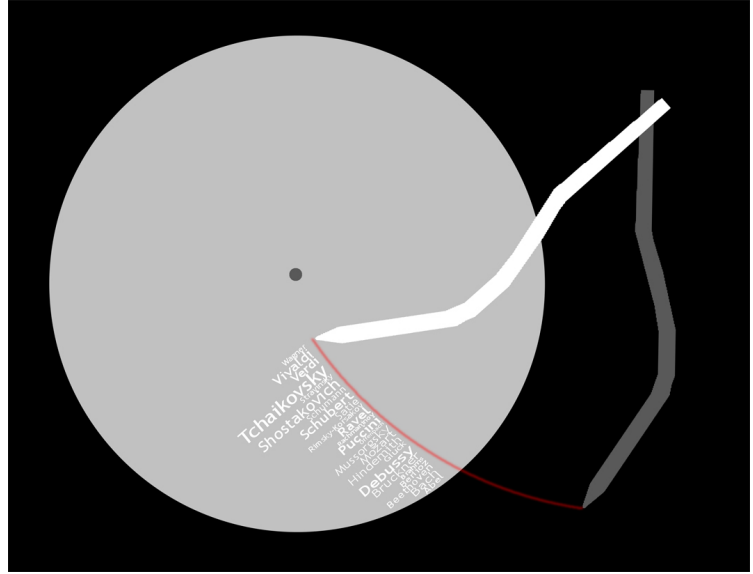

Figure 3: Tag cloud positioning navigation

This can be further enhanced by projecting a visual cue onto the turntable. As can be seen in figure 3 we project an alphabetical tag cloud alongside the line the tone arm can be set to. Navigation based on tag clouds [2] has become quite popular in recent Web 2.0 applications where a hierarchical navigation is as inapplicable as in music organization. Concepts are visually accentuated according to their frequency of occurrence. As there is no space in between them, the font size of the tags is proportional to their occurrence frequency. A tag that happens to occur ten times will be displayed using a font size five times as big as one that occurs only twice. In this idealized set-up the visual cue astonishingly happens to remain perfectly in sync with the music collection all the time. To ensure perceptibility of the projected tags, we had to introduce a certain threshold of frequency though and leave out the least frequent ones. This gives rise to a slight distortion of the visual cue that can be tolerated. Compared to a pure browsing system without any direct positioning at all, the search performance can be increased by a factor equivalent to the number of perceivable tags for a worst case scenario.

\section{Scratching gestures to switch criteria}

There can only be one sorting criterion at a time, but a DJ might recall a different aspect of the record than the one displayed. In this case, the whole positioning would be rendered useless. This urges us to provide a mechanism of switching criteria for the music collection sorting and respective tag cloud display. In analogy to mouse gestures [4] we propose simple scratching gestures to issue the switching command. A feasible first approach would be to detect double baby scratches (forward backward forward backward) [3] during browsing and trigger a switching of criteria in round robin manner. One can even think of more advanced individual scratch gestures for every single criterion, so that a progressive filtering (style: Pop/Rock, artist: Wagner) would further speed up the search task.

\section{Conclusion}

We understand TIMBAP as a complete digital DJing system with a substantially improved user interface. It can be installed with little effort at a venue and is accessible to any DJ with a mobile storage device. Our goal is to finally make a change-over of DJs as simple as unplugging one USB drive and plugging in another one. At the same time, TIMBAP is also appealing to hobby djs on a low budget. The costly projection can be substituted with a normal display screen nearby. We claim that the physical user interface becomes second nature quite quickly to anyone without any prior knowledge. Therefore and because its hardware is so much more robust compared to other tangible interfaces, the demonstration system shall be open for evaluation to every participant of the conference.

\section{Acknowledgements}

TIMBAP uses MsPinky objects for timecode processing. We want to thank the MsPinky developers for making their interface readily accessible.

\section{References}

[1] T. H. Andersen. Mixxx: towards novel dj interfaces. In NIME '03: Proceedings of the 2003 conference on New interfaces for musical expression, pages 30-35, Singapore, 2003. National University of Singapore.

[2] Jenneke Fokker, Johan Pouwelse, and Wray Buntine. Tag-based navigation for peer-to-peer wikipedia. In Collaborative Web Tagging Workshop held in conjuction with the 15th International World Wide Web Conference (WWW2006), page CDROM, May 2006.

[3] K. F. Hansen. The basics of Scratching. Journal of New Music Research, 31(4):357-367, 2002.

[4] M. Karam and M. C. Schraefel. A taxonomy of gestures in human computer interactions. Technical report, University of Southampton, November 2005.

[5] S. R. Klemmer, B. Hartmann, and L. Takayama. How bodies matter: five themes for interaction design. In DIS '06: Proceedings of the 6th ACM conference on Designing Interactive systems, pages 140-149, New York, NY, USA, 2006. ACM Press.

[6] Takuro Mizuta Lippit. Turntable music in the digital era: Designing alternative tools for new turntable expression. In NIME '06: Proceedings of the 2006 conference on New interfaces for musical expression, pages 71-74. IRCAM - Centre Pompidou in collaboration with Sorbonne University, 2006.

[7] N. Reithinger, D. Fedeler, A. Kumar, C. Lauer, E. Pecourt, and L. Romary. Miamm - a multimodal dialogue system using haptics. In J. van Kuppevelt, L. Dybkjaer and N. O. Bernsen, editors, Advances in Natural Multimodal Dialogue Systems, pages 307-332, Dordrecht, Netherlands, 2005. Springer. 